\title{
THE LATTICE OF FITTING CLASSES WHICH ARE RIGHT EXTENSIBLE BY SOLUBLE GROUPS
}

\author{
M. J. Iranzo*, Julio P. Lafuente ${ }^{\dagger}$ and F. PÉrez-Monasor* \\ In memory of Professor Klaus Doerk
}

Abstract

In this paper we study the set of Fitting classes which are right extensible by soluble groups ordered by the inclusion relation. The consideration of the associated lattices gives rise to new Fitting classes and it allows to obtain some injectivity criteria for general Fitting classes.

All considered groups are assumed to be finite. Set

$$
\mathcal{T}=\{\mathfrak{T} ; \mathfrak{T} \text { is a Fitting class such that } \mathfrak{T}=\mathfrak{T} \mathfrak{S}\},
$$

where $\mathfrak{S}$ is the class of soluble groups. The classes in $\mathfrak{T}$ have a particular interest in the Theory of Fitting classes of finite groups which are not necessarily soluble. For instance, in a previous paper the authors show that, if $\mathfrak{T} \in \mathcal{T}$, then each intermediate Fitting class $\mathfrak{F}$ between $\mathfrak{T}_{*}$ and $\langle\mathfrak{T}, \mathrm{b}(\mathfrak{T})\rangle$ is injective (that is each group has $\mathfrak{F}$-injectors) -in particular, each Fitting class in Locksec(S) is injective, in accordance with a wider conjecture of Shemetkov $[4,11.117]$ - , being normal Fitting classes precisely those belonging to $\operatorname{Locksec}(\langle\mathfrak{T}, \mathrm{b}(\mathfrak{T})\rangle)$.

In this paper we study the set $\mathcal{T}$ ordered by the inclusion relation. The consideration of the lattices associated to $\mathcal{T}$ gives rise to new Fitting classes, as the complement in $\mathcal{X}_{\mathfrak{N}}$ of the class of generalized nilpotent groups and the complement in $\mathcal{T}_{\mathfrak{N}}$ of the class of nilpotent-constrained groups.

The notations and terminology are referred to [1]. We denote by $\mathfrak{E}$ the class of all (finite) groups. Recall that a class of groups $\mathfrak{F}$ is a Fitting class if (i) $N \unlhd G \in \mathfrak{F}$ implies $N \in \mathfrak{F}$, and (ii) $N, M \unlhd G \in \mathfrak{E}$,

2000 Mathematics Subject Classification. 20D10.

Key words. Finite group, Fitting class, lattice, preboundary.

*Supported by MTM2004-06067-C02-01.

†Supported by MTM2004-08219-C02-01. 
$N, M \in \mathfrak{F}$ implies $N M \in \mathfrak{F}$. If $\mathfrak{F}$ is a class of groups, the product $G_{\mathfrak{F}}$ of all normal subgroups of $G \in \mathfrak{E}$ which belong to $\mathfrak{F}$ is called the radical of $G$ with respect to $\mathfrak{F}$. The Fitting product of two Fitting classes $\mathfrak{F}$ and $\mathfrak{G}$ is $\mathfrak{F} \diamond \mathfrak{G}=\left(G \in \mathfrak{E} ; G / G_{\mathfrak{F}} \in \mathfrak{G}\right)$. The product of two classes of groups $\mathfrak{F}$ and $\mathfrak{G}$ is the class $\mathfrak{F} \mathfrak{G}$ of the groups $G \in \mathfrak{E}$ which have a normal subgroup $N$ such that $N \in \mathfrak{F}$ and $G / N \in \mathfrak{G}$. For a set of groups $\mathrm{X}$, $\langle X\rangle$ denotes the smallest Fitting class containing $X$. The boundary of the Fitting class $\mathfrak{F}$ is the set $\mathrm{b}(\mathfrak{F})$ of the groups $X$ such that $X \notin \mathfrak{F}$ and $N \operatorname{sn} G, N \neq G$ implies $N \in \mathfrak{F}$ (where $N \operatorname{sn} G$ means that $N$ is a subnormal subgroup of $G$ ). Each group $X \in \mathrm{b}(\mathfrak{F})$ is single-headed (that is $X$ has a unique maximal normal subgroup, denoted $\operatorname{Cos}(X))$ and $\mathrm{b}(\mathfrak{F})$ is subnormally independent (that is $X \operatorname{sn} Y \in \mathrm{b}(\mathfrak{F}), X \neq Y$ implies $X \notin \mathrm{b}(\mathfrak{F}))$. A group $G \in \mathfrak{E}$ is perfect if $G=G^{\prime} . \overline{\mathrm{b}}(\mathfrak{F})$ is the set of all perfect groups $X \in \mathrm{b}(\mathfrak{F})$. Set $\mathfrak{F}^{b}=\langle\operatorname{Cos}(X) ; X \in \mathrm{b}(\mathfrak{F})\rangle, \mathfrak{F}^{m}=\langle\mathrm{b}(\mathfrak{F})\rangle$ and $\mathfrak{F}^{\mathfrak{s}}=\left\langle G \in \mathfrak{F} ; G^{\prime}=G\right\rangle$. If $\mathbf{X}$ is a class of groups, $\mathbf{D}_{0} \mathrm{X}$ denotes the class of all groups which are a direct product of elements of $X$. If $\mathfrak{F} \subseteq \mathfrak{X}$ are Fitting classes, we set $\mathfrak{X}_{\mathfrak{F}}=\left(G \in \mathfrak{X} ; G / G_{\mathfrak{F}} \in \mathbf{D}_{0} \Sigma\right)$, where $\Sigma$ is the class of all nonabelian simple groups.

A class $\mathfrak{m}$ of groups is a preboundary if it is subnormally independent and consists of single-headed groups. Denote by $\mathcal{P}$ the set of all preboundaries of perfect groups. If $\mathfrak{m} \in \mathcal{P}$ and $G \in \mathfrak{E}$, set $\mathrm{b}_{\mathfrak{m}}(G)=$ $\{X \operatorname{sn} G ; X \in \mathfrak{m}\}$. The map b: $\mathcal{T} \rightarrow \mathcal{P}$ is a bijection whose inverse $\mathrm{h}: \mathcal{P} \rightarrow \mathcal{T}$ is given by $\mathrm{h}(\mathfrak{m})=\left(G \in \mathfrak{E} ; \mathrm{b}_{\mathfrak{m}}(G)=\emptyset\right)$ if $\mathfrak{m} \in \mathcal{P}[\mathbf{1}$, XI 4.4].

We begin with

Theorem 1. $(\mathcal{T}, \subseteq)$ with the operations

$$
\mathfrak{T} \wedge \mathfrak{R}=\mathfrak{T} \cap \mathfrak{R}, \quad \mathfrak{T} \vee \mathfrak{R}=\langle\mathfrak{T}, \mathfrak{R}\rangle \mathfrak{S},
$$

if $\mathfrak{T}, \mathfrak{R} \in \mathcal{T}$, is a complete, distributive and atomic lattice. $\mathcal{T}$ has no coatoms.

Proof: It is immediate that $(\mathfrak{T} \cap \mathfrak{R}) \mathfrak{S}=\mathfrak{T} \cap \mathfrak{R}$, and therefore $(\mathcal{T}, \subseteq)$ is a complete lattice.

Denote by $\vee$ and $\wedge$ the corresponding lattice operations. We have that, if $\mathfrak{T}, \mathfrak{R} \in \mathcal{T}$, then $\mathfrak{T} \wedge \mathfrak{R}=\mathfrak{T} \cap \mathfrak{R}$. And immediatly $\mathfrak{T} \vee R=\langle\mathfrak{T}, \mathfrak{R}\rangle \mathfrak{S}$.

Let us see that the lattice is distributive. We must show that, if $\mathfrak{T}, \mathfrak{R}, \mathfrak{K} \in \mathcal{T}$, then

$$
\mathfrak{T} \cap \mathfrak{F} \mathfrak{S}=\mathfrak{H} \mathfrak{S},
$$

where $\mathfrak{F}=\langle\mathfrak{R}, \mathfrak{K}\rangle$ and $\mathfrak{H}=\langle\mathfrak{T} \cap \mathfrak{R}, \mathfrak{T} \cap \mathfrak{K}\rangle$. As $\mathfrak{T} \cap \mathfrak{F} \mathfrak{S}=(\mathfrak{T} \cap \mathfrak{F}) \mathfrak{S}$, this is equivalent to

$$
(\mathfrak{T} \cap \mathfrak{F}) \mathfrak{S}=\mathfrak{H} \mathfrak{S} .
$$


Clearly $\mathfrak{H} \subseteq \mathfrak{T} \cap \mathfrak{F}$. Let us assume that $\mathfrak{H} \mathfrak{S} \neq(\mathfrak{T} \cap \mathfrak{F}) \mathfrak{S}$. Then there exists

$$
X \in(\mathfrak{T} \cap \mathfrak{F}) \mathfrak{S} \cap \mathrm{b}(\mathfrak{H} \mathfrak{S}) .
$$

$X$ is a perfect and single-headed group, hence $X \in \mathfrak{T} \cap \mathfrak{F}$. As $\mathfrak{F}=\langle\mathfrak{R}, \mathfrak{K}\rangle$, $X \in \mathbf{S}_{n} \mathfrak{R} \cup \mathbf{S}_{n} \mathfrak{K}$ by $[\mathbf{1}$, XI 4.14]. Therefore $X \in(\mathfrak{T} \cap \mathfrak{R}) \cup(\mathfrak{T} \cap \mathfrak{K}) \subseteq \mathfrak{H}$, a contradiction.

The lattice is atomic as a particular case of Proposition 4 and has no coatoms as a consequence of Proposition 7.

Remarks 2. i) If $\mathfrak{T}, \mathfrak{R} \in \mathcal{T}$ and $\mathfrak{T} \neq \mathfrak{E} \neq \mathfrak{R}$, then $\langle\mathfrak{T}, \mathfrak{R}\rangle \mathfrak{S} \neq \mathfrak{E}$. In particular, the unique elements of $\mathcal{T}$ which have a complement are $\mathfrak{S}$ and $\mathfrak{E}$.

Suppose, to have a contradiction, that $\langle\mathfrak{T}, \mathfrak{R}\rangle \mathfrak{S}=\mathfrak{E}$. Let $X \in \mathrm{b}(\mathfrak{T})$, $Y \in \mathrm{b}(\mathfrak{R})$ and take $S \in \Sigma$. Then the regular wreath product $W=$ $(X \times Y) \imath S$ is perfect and single-headed, by [1, A 18.8]. By hypothesis, $W \in\langle\mathfrak{T}, \mathfrak{R}\rangle$, hence either $W \in \mathfrak{T}$ or $W \in \mathfrak{R}$, by [1, XI 4.14], against $X \notin \mathfrak{T}$ and $Y \notin \mathfrak{R}$.

ii) Let $\mathfrak{T}, \mathfrak{R} \in \mathcal{T}, \mathfrak{T} \nsubseteq \mathfrak{R} \nsubseteq \mathfrak{T}$. Then $\langle\mathfrak{T}, \mathfrak{R}\rangle$ is strictly contained in $\mathfrak{T} \vee \mathfrak{R}$.

To see it, consider

$$
\mathfrak{H}=\left(G \in \mathfrak{E} ; G /\left(G_{\mathfrak{T}} G_{\mathfrak{R}}\right) \in \mathfrak{N}\right) .
$$

By $[\mathbf{1}$, IX 2.1], $\mathfrak{H}$ is a Fitting class which contains both $\mathfrak{T}$ and $\mathfrak{R}$. Let us assume that $\mathfrak{G} \in \mathcal{T}$ is such that $\mathfrak{T}, \mathfrak{R} \subseteq \mathfrak{G}$. Let $G \in \mathfrak{H}$. Then $G_{\mathfrak{T}} G_{\mathfrak{R}} \in \mathfrak{G}$ and $G /\left(G_{\mathfrak{T}} G_{\mathfrak{R}}\right) \in \mathfrak{N}$. Thus $G \in \mathfrak{G} \mathfrak{S}=\mathfrak{G}$. Therefore $\mathfrak{H} \subseteq \mathfrak{T} \vee \mathfrak{R}$.

Let us take $R \in \mathfrak{R} \cap \mathrm{b}(\mathfrak{T})$ and $T \in \mathfrak{T} \cap \mathrm{b}(\mathfrak{R})$ and form the regular wreath product $G=(T \times R)$ 乙 $S_{3}$. By [1, X 1.15], $\mathfrak{T}$ and $\mathfrak{R}$ are Lockett classes. Then, by $[\mathbf{1}, \mathrm{X} 2.1], G /\left(G_{\mathfrak{T}} G_{\mathfrak{R}}\right) \cong S_{3} \notin \mathfrak{N}$. Therefore $G \notin \mathfrak{H}$. But $G$ has a subgroup $H$ such that $H /\left(H_{\mathfrak{T}} H_{\mathfrak{R}}\right) \cong C_{3} \in \mathfrak{N}$ and $G / H \cong$ $C_{2}$, that is $G \in \mathfrak{H} \mathfrak{S}$. In particular $G \in(\mathfrak{T} \vee \mathfrak{R}) \backslash\langle\mathfrak{T}, \mathfrak{R}\rangle$.

If $\mathfrak{X}$ and $\mathfrak{Y}$ are classes of groups, we set

$$
\mathfrak{X} \cdot \mathfrak{Y}=\left(G \in \mathfrak{E} ; G=G_{\mathfrak{X}} G_{\mathfrak{Y}}\right) .
$$

Notice that if $\mathfrak{X}$ and $\mathfrak{Y}$ are Fitting classes, not necessarily $\mathfrak{X} \cdot \mathfrak{Y}$ is a Fitting class (see $[\mathbf{1}$, Remark (b) after IX 2.1]). If $\mathfrak{m} \in \mathcal{P}$, we write

$$
\mathrm{E}_{\mathfrak{m}}(G)=\left\langle X ; X \in \mathrm{b}_{\mathfrak{m}}(G)\right\rangle .
$$

Lemma 3. Let $\mathfrak{F}$ be a Fitting class and $\mathfrak{n} \subseteq \overline{\mathrm{b}}(\mathfrak{F})$. Then we have:

$$
\langle\mathfrak{F}, \mathfrak{n}\rangle=\mathfrak{F} \cdot\langle\mathfrak{n}\rangle=\left(G \in \mathfrak{E} ; G=G_{\mathfrak{F}} \mathrm{E}_{\mathfrak{n}}(G)\right) .
$$


Proof: Let $G \in \mathfrak{E}$. If $X \in \mathrm{b}_{\mathfrak{n}}(G)$, then $\operatorname{Cos}(X)=X_{\mathfrak{F}}$.

Set $\mathfrak{X}=\left(G \in \mathfrak{E} ; G=G_{\mathfrak{F}} \mathrm{E}_{\mathfrak{n}}(G)\right)$. Let us see that it is a Fitting class.

If $G \in \mathfrak{X}$, then $G / G_{\mathfrak{F}} \cong \mathrm{E}_{\mathfrak{n}}(G) / \mathrm{E}_{\mathfrak{n}}(G)_{\mathfrak{F}} \in \mathbf{D}_{0} \Sigma$ [2, Lemma 6]. Let $N \unlhd G$. Then $\mathrm{b}_{\mathfrak{n}}(N) \subseteq \mathrm{b}_{\mathfrak{n}}(G)$. Thus, if $\mathrm{b}_{\mathfrak{n}}(N)=\left\{X_{1}, \ldots, X_{r}\right\}$, then

$$
N G_{\mathfrak{F}} / G_{\mathfrak{F}}=X_{1} G_{\mathfrak{F}} / G_{\mathfrak{F}} \times \cdots \times X_{r} G_{\mathfrak{F}} / G_{\mathfrak{F}},
$$

hence

$N=N \cap N G_{\mathfrak{F}}=N \cap X_{1} \ldots X_{r} G_{\mathfrak{F}}=N \cap \mathrm{E}_{\mathfrak{n}}(N) G_{\mathfrak{F}}=\mathrm{E}_{\mathfrak{n}}(N) N_{\mathfrak{F}} \in \mathfrak{X}$.

On the other side, if $N, M \unlhd G, G=N M, N, M \in \mathfrak{X}$, then

$$
G=N M=N_{\mathfrak{F}} \mathrm{E}_{\mathfrak{n}}(N) M_{\mathfrak{F}} \mathrm{E}_{\mathfrak{n}}(M) \leq G_{\mathfrak{F}} \mathrm{E}_{\mathfrak{n}}(G),
$$

hence $G \in \mathfrak{X}$. Therefore $\mathfrak{X}$ is a Fitting class.

Obviously $\mathfrak{X} \subseteq\langle\mathfrak{F}, \mathfrak{n}\rangle$, hence $\mathfrak{X}=\langle\mathfrak{F}, \mathfrak{n}\rangle$.

Set $\mathfrak{L}=\langle\mathfrak{n}\rangle$. As $\mathrm{E}_{\mathfrak{n}}(G) \leq G_{\mathfrak{L}}$, we have $G_{\mathfrak{F}} \mathrm{E}_{\mathfrak{n}}(G) \leq G_{\mathfrak{F}} G_{\mathfrak{L}}$ for each $G \in \mathfrak{E}$. As $\mathfrak{L}=\langle\mathfrak{n}\rangle \subseteq\langle\mathfrak{F}, \mathfrak{n}\rangle=\mathfrak{X}$, we have

$$
G_{\mathfrak{F}} G_{\mathfrak{L}} \leq G_{\mathfrak{F}} \mathrm{E}_{\mathfrak{n}}(G) \leq G_{\mathfrak{F}} G_{\mathfrak{L}},
$$

hence $G_{\mathfrak{F}} \mathrm{E}_{\mathfrak{n}}(G)=G_{\mathfrak{F}} G_{\mathfrak{L}}$.

Proposition 4. If $\mathfrak{E} \neq \mathfrak{T} \in \mathcal{T}$, then

$$
[\mathfrak{T}, \rightarrow)=\{\mathfrak{H} \in \mathcal{T} ; \mathfrak{T} \subseteq \mathfrak{H}\}
$$

is atomic. Moreover there is a bijective correspondence between the set of atoms of $[\mathfrak{T}, \rightarrow)$ and $\mathrm{b}(\mathfrak{T})$.

Proof: Let us see that $\mathfrak{H} \in \mathcal{T}$ is an atom in $[\mathfrak{T}, \rightarrow)$ if and only if $\mathfrak{H}=$ $\langle\mathfrak{T}, X\rangle \mathfrak{S}$, where $X \in \mathrm{b}(\mathfrak{T})$.

Assume that $\mathfrak{H}$ is an atom in $[\mathfrak{T}, \rightarrow)$. Let $X \in \mathfrak{H} \cap \mathrm{b}(\mathfrak{T})$. Then

$$
\mathfrak{T} \subset\langle\mathfrak{T}, X\rangle \mathfrak{S} \subseteq \mathfrak{H}
$$

hence $\mathfrak{H}=\langle\mathfrak{T}, X\rangle \mathfrak{S}$. Assume conversely that $X \in \mathrm{b}(\mathfrak{T}), \mathfrak{H} \in \mathcal{T}$ and

$$
\mathfrak{T} \subset \mathfrak{H} \subseteq\langle\mathfrak{T}, X\rangle \mathfrak{S} .
$$

Take $Y \in \mathrm{b}(\mathfrak{T}) \cap \mathfrak{H}$. As $Y$ is perfect, then $Y \in\langle\mathfrak{T}, X\rangle$. Then $Y \in \mathbf{S}_{n} X$, by $[\mathbf{1}, \mathrm{XI} 4.14]$, hence $Y \cong X$ because $\operatorname{Cos}(X) \in \mathfrak{T}$. Therefore $\mathfrak{H}=$ $\langle\mathfrak{T}, X\rangle \mathfrak{S}$.

Now if $\mathfrak{T} \subset \mathfrak{H} \in \mathcal{T}$, let $X \in \mathrm{b}(\mathfrak{T}) \cap \mathfrak{H}$. Then $\langle\mathfrak{T}, X\rangle \mathfrak{S} \subseteq \mathfrak{H}$, hence $[\mathfrak{T}, \rightarrow)$ is atomic.

Finally we can show as above that if $X, Y \in \mathrm{b}(\mathfrak{T})$, then $\langle\mathfrak{T}, X\rangle \mathfrak{S}=$ $\langle\mathfrak{T}, Y\rangle \mathfrak{S}$ if and only if $X \cong Y$. 
The classes $\mathfrak{T} \in \mathcal{T}$ for which $\mathrm{b}(\mathfrak{T})$ is maximal in $(\mathcal{P}, \subseteq)$, where $\mathcal{P}$ is the set of all preboundaries of perfect groups, are precisely those with $\mathfrak{T}^{b}=\mathfrak{T}_{*}[\mathbf{2}$, Proposition 13]. We have

Proposition 5. Let $\mathfrak{E} \neq \mathfrak{T} \in \mathcal{T}$. If $\mathfrak{H}$ is an atom in $[\mathfrak{T}, \rightarrow)$, then $\mathfrak{H}^{b}=\mathfrak{H}_{*}$.

Proof: By Proposition 4, $\mathfrak{H}=\langle\mathfrak{T}, X\rangle \mathfrak{S}$, where $X \in \mathrm{b}(\mathfrak{T})$. Now if $Y \in \mathfrak{H}$ is perfect and single-headed, then either $Y \in \mathfrak{T}$ or $Y \cong X$, by [1, XI 4.14], hence $\mathfrak{H}^{\mathfrak{s}}=\left\langle\mathfrak{T}^{\mathfrak{s}}, X\right\rangle$.

On the other hand, if $S \in \Sigma$, then $X \imath S \in \mathrm{b}(\mathfrak{H})$, because $X$ is perfect and single-headed by $[\mathbf{1}, \mathrm{A} 18.8(\mathrm{~d})]$, hence $X \in \mathfrak{H}^{b}$.

We proceed now to show that $\mathfrak{T}^{\mathfrak{s}} \subseteq \mathfrak{H}^{b}$. Let $Y \in \mathfrak{T}$ perfect and single-headed and take $G=X \times Y$. Let $S \in \Sigma$ and $W=G \imath S$. $W$ is perfect and single-headed again by $[\mathbf{1}, \mathrm{A} 18.8(\mathrm{~d})]$. Assume that $W \in \mathfrak{H}$. Then $W \in\langle\mathfrak{T}, X\rangle$, hence either $W \in \mathfrak{T}$ or $W \operatorname{sn} X$, by [1, XI 4.14], a contradiction. As $\operatorname{Cos}(W) \in \mathfrak{H}$, we have that $W \in \mathrm{b}(\mathfrak{H})$. Therefore $\operatorname{Cos}(W) \in \mathfrak{H}^{b}$, hence $Y \in \mathfrak{H}^{b}$. And $\mathfrak{T}^{\mathfrak{s}} \subseteq \mathfrak{H}^{b}$.

Finally

by $[3,1.1]$.

$$
\mathfrak{H}_{*}=\left\langle\mathfrak{H}^{\mathfrak{s}}, \mathfrak{H}^{b}\right\rangle=\left\langle\mathfrak{T}^{\mathfrak{s}}, X, \mathfrak{H}^{b}\right\rangle=\mathfrak{H}^{b}
$$

Remark 6. As a consequence of Proposition 5 we have that $\left\{\mathfrak{T} \in \mathcal{T} ; \mathfrak{T}^{b}=\right.$ $\left.\mathfrak{T}_{*}\right\}$ is not a sublattice of $\mathcal{T}$. Take any $\mathfrak{T} \in \mathcal{T}$ such that $\mathfrak{T}^{b} \neq \mathfrak{T}_{*}$ and $\mathfrak{T} \neq \mathfrak{E}$ (for instance the class $\mathfrak{C}$ of $\mathfrak{N}$-constrained groups, see $[\mathbf{2}$, Example (iii)]) and two different atoms $\mathfrak{H}, \mathfrak{K} \in[\mathfrak{T}, \rightarrow)$. Then $\mathfrak{H}^{b}=\mathfrak{H}_{*}$ and $\mathfrak{K}^{b}=\mathfrak{K}_{*}$, by Proposition 5 , but $(\mathfrak{H} \cap \mathfrak{K})^{b}=\mathfrak{T}^{b} \neq \mathfrak{T}_{*}=(\mathfrak{H} \cap \mathfrak{K})_{*}$.

Proposition 7. Let $\mathfrak{S} \neq \mathfrak{H} \in \mathcal{T}$. Then the set of coatoms of

$$
(\leftarrow, \mathfrak{H}]=\{\mathfrak{G} \in \mathcal{T} ; \mathfrak{G} \subseteq \mathfrak{H}\}
$$

is in bijective correspondence with the maximal elements of

$$
\operatorname{psh}(\mathfrak{H})=(X \in \mathfrak{H} ; X \text { is perfect and single-headed })
$$

ordered by the relation "to be subnormally embedded".

Proof: Let us assume that $\mathfrak{T}$ is a coatom in $(\leftarrow, \mathfrak{H}]$. Then, if $X \in \mathfrak{H} \cap$ $\mathrm{b}(\mathfrak{T})$, we have $\mathfrak{H}=\langle\mathfrak{T}, X\rangle \mathfrak{S}$. If $Y$ is a perfect and single-headed group in $\mathfrak{H}$ and $X \operatorname{sn} Y$, then $X \cong Y$, by [1, XI 4.14], and $X$ is maximal. Further if $Z \in \mathfrak{H} \cap \mathrm{b}(\mathfrak{T})$, then

$$
\langle\mathfrak{T}, X\rangle \mathfrak{S}=\mathfrak{H}=\langle\mathfrak{T}, Z\rangle \mathfrak{S} .
$$

Therefore $X \in\langle\mathfrak{T}, Z\rangle \mathfrak{S}$, hence $X \operatorname{sn} Z$ and $X \cong Z$ by the maximality of $X$. Thus $\mathfrak{H} \cap \mathrm{b}(\mathfrak{T})=(X)$. 
Assume now that $X$ is a maximal element in $\operatorname{psh}(\mathfrak{H})$. Consider

$$
\mathfrak{m}=(\mathrm{b}(\mathfrak{H}) \backslash(Y ; X \operatorname{sn} Y)) \cup(X) .
$$

Clearly $\mathfrak{m} \in \mathcal{P}$. Let $\mathfrak{T}=\mathrm{h}(\mathfrak{m})$. Let us see that $\mathfrak{H}=\langle\mathfrak{T}, X\rangle \mathfrak{S}$. Suppose that there exists $Y \in \mathfrak{T} \cap \mathrm{b}(\mathfrak{H})$. As $\mathrm{b}(\mathfrak{T})=\mathfrak{m}$, it follows that $X \operatorname{sn} Y \in$ $\mathrm{b}(\mathfrak{H})$, hence $X \in \mathfrak{T}$, a contradiction. Therefore $\mathfrak{T} \subseteq \mathfrak{H}$, hence $\langle\mathfrak{T}, X\rangle \mathfrak{S} \subseteq$ $\mathfrak{H}$. Suppose $Y \in \mathfrak{H} \cap \mathrm{b}(\langle\mathfrak{T}, X\rangle \mathfrak{S}) . Y$ is a perfect and single-headed group; by the maximality of $X$ we have that $X$ is not subnormally embedded in $Y$. Therefore, if $Z \operatorname{sn} Y$, then $Z \notin \mathfrak{m}$. Thus $Y \in \mathrm{h}(\mathfrak{m})=\mathfrak{T}$, a contradiction. So $\mathfrak{H}=\langle\mathfrak{T}, X\rangle \mathfrak{S}$ and $\mathfrak{T}$ is a coatom in $(\leftarrow, \mathfrak{H}]$.

Corollary 8. If $\mathfrak{T} \in \mathcal{T}$ is such that $\mathfrak{T}^{b} \neq \mathfrak{T}_{*}$, then each perfect and single-headed group in $\mathfrak{T}$ is strictly subnormally embedded in another perfect and single-headed group in $\mathfrak{T}$.

Proof: Assume the contrary. Then psh(T) has maximal elements for the relation "to be subnormally embedded", hence there exists a coatom $\mathfrak{K}$ in $(\leftarrow, \mathfrak{T}]$, by Proposition 7. But then $\mathfrak{T}$ is an atom in $[\mathfrak{K}, \rightarrow)$, hence $\mathfrak{T}^{b}=\mathfrak{T}_{*}$ by Proposition 5 , against the hypothesis.

We know that $\mathfrak{T} \in \mathcal{T}$ if and only if there exist Fitting classes $\mathfrak{F}$ and $\mathfrak{X}$ such that $\mathfrak{F} \subseteq \mathfrak{X} \subseteq \mathfrak{X}_{\mathfrak{F}}$ with $\mathfrak{T}=\mathfrak{T}(\mathfrak{F}, \mathfrak{X})$, where $\mathfrak{T}(\mathfrak{F}, \mathfrak{X})=\left(G \in \mathfrak{E} ; G_{\mathfrak{X}} \in\right.$ $\mathfrak{F})[\mathbf{2}$, Theorem 8]. We set $\mathcal{B}(X)$ for the boolean of $X$.

Lemma 9. If $\mathfrak{F}$ is a Fitting class, we write

$$
\mathcal{X}_{\mathfrak{F}}=\left\{\mathfrak{X} ; \mathfrak{X} \text { is a Fitting class such that } \mathfrak{F} \subseteq \mathfrak{X}=\mathfrak{X}_{\mathfrak{F}}\right\} \text {. }
$$

Then

i) The map

$$
\mathrm{f}: \mathcal{B}(\overline{\mathrm{b}}(\mathfrak{F})) \rightarrow \mathcal{X}_{\mathfrak{F}}
$$

given by $\mathrm{f}(\mathfrak{m})=\langle\mathfrak{F}, \mathfrak{m}\rangle$ if $\mathfrak{m} \subseteq \overline{\mathrm{b}}(\mathfrak{F})$, is an isomorphism of inclusionordered sets.

ii) $\left(\mathcal{X}_{\mathfrak{F}}, \subseteq\right)$ with the operations

$$
\mathfrak{X} \wedge \mathfrak{Y}=\mathfrak{X} \cap \mathfrak{Y}, \quad \mathfrak{X} \vee \mathfrak{Y}=\mathfrak{X} \cdot \mathfrak{Y},
$$

if $\mathfrak{X}, \mathfrak{Y} \in \mathcal{X}_{\mathfrak{F}}$, is a complete, distributive, complemented and atomic lattice.

Proof: i) Let us see that, if $\mathfrak{F}$ is a Fitting class and $\mathfrak{F} \subseteq \mathfrak{X}$, then $\mathfrak{X} \in \mathcal{X}_{\mathfrak{F}}$ if and only if $\mathfrak{X}=\langle\mathfrak{F}, \mathfrak{X} \cap \overline{\mathrm{b}}(\mathfrak{F})\rangle$. Set $\mathfrak{m}=\mathfrak{X} \cap \overline{\mathrm{b}}(\mathfrak{F})$.

If $\mathfrak{X}=\langle\mathfrak{F}, \mathfrak{m}\rangle$, then $G \in \mathfrak{X}$ implies $G=G_{\mathfrak{F}} \mathrm{E}_{\mathfrak{m}}(G)$, by Lemma 3 , hence $G / G_{\mathfrak{F}} \cong \mathrm{E}_{\mathfrak{m}}(G) / \mathrm{E}_{\mathfrak{m}}(G)_{\mathfrak{F}} \in \mathbf{D}_{0} \Sigma$. Therefore $\mathfrak{X}=\mathfrak{X}_{\mathfrak{F}}$.

Assume conversely that $\mathfrak{X} \in \mathcal{X}_{\mathfrak{F}}$. Obviously $\langle\mathfrak{F}, \mathfrak{m}\rangle \subseteq \mathfrak{X}$. Let $G \in \mathfrak{X}$. As $\mathfrak{X}=\mathfrak{X}_{\mathfrak{F}}$, we have $G / G_{\mathfrak{F}} \in \mathbf{D}_{0} \Sigma$. We may assume that $G \notin \mathfrak{F}$. 
Let $N / G_{\mathfrak{F}}$ be a minimal normal subgroup of $G / G_{\mathfrak{F}}$. Take $X \operatorname{sn} G$ a minimal supplement of $G_{\mathfrak{F}}$ in $N$. Then $X$ is perfect and single-headed and $\operatorname{Cos}(X)=X_{\mathfrak{F}}$, hence $X \in \overline{\mathrm{b}}(\mathfrak{F}) \cap \mathfrak{X}=\mathfrak{m}$. It follows that $G=$ $G_{\mathfrak{F}} \mathrm{E}_{\mathfrak{m}}(G) \in\langle\mathfrak{F}, \mathfrak{m}\rangle$, and $\mathfrak{X} \subseteq\langle\mathfrak{F}, \mathfrak{m}\rangle$.

Therefore $\mathrm{f}$ is a well defined bijection between the boolean of $\overline{\mathrm{b}}(\mathfrak{F})$ and $\mathcal{X}_{\mathfrak{F}}$. Let $\mathfrak{m}, \mathfrak{n} \subseteq \overline{\mathrm{b}}(\mathfrak{F})$. If $\mathfrak{m} \subseteq \mathfrak{n}$, then obviously $\mathrm{f}(\mathfrak{m}) \subseteq \mathrm{f}(\mathfrak{n})$. Finally, assume that $\langle\mathfrak{F}, \mathfrak{m}\rangle \subseteq\langle\mathfrak{F}, \mathfrak{n}\rangle$. Let $X \in \mathfrak{m}$. Then $X \in\langle\mathfrak{F}, \mathfrak{n}\rangle$, hence $X \in \mathfrak{n}$ by $[\mathbf{1}, \mathrm{XI} 4.14]$, as $\mathfrak{n}$ is subnormally independent.

ii) It suffices to show that, if $\mathfrak{m}, \mathfrak{n} \subseteq \overline{\mathrm{b}}(\mathfrak{F}), \mathfrak{X}=\langle\mathfrak{F}, \mathfrak{m}\rangle$ and $\mathfrak{Y}=\langle\mathfrak{F}, \mathfrak{n}\rangle$, then $\mathfrak{X} \cdot \mathfrak{Y}=\langle\mathfrak{F}, \mathfrak{m} \cup \mathfrak{n}\rangle$. Now $G \in \mathfrak{X} \cdot \mathfrak{Y} \Longleftrightarrow G=G_{\mathfrak{X}} G_{\mathfrak{Y}} \Longleftrightarrow$ $G=G_{\mathfrak{F}} \mathrm{E}_{\mathfrak{m}}(G) \mathrm{E}_{\mathfrak{n}}(G)$ (by Lemma 3$) \Longleftrightarrow G \in\langle\mathfrak{F}, \mathfrak{m} \cup \mathfrak{n}\rangle$, as obviously $\mathrm{E}_{\mathfrak{m}}(G) \mathrm{E}_{\mathfrak{n}}(G)=\mathrm{E}_{\mathfrak{m} \cup \mathfrak{n}}(G)$.

Theorem 10. Let $\mathfrak{F}$ be a Fitting class and set

$$
\mathcal{T}_{\mathfrak{F}}=\left\{\mathfrak{T} \in \mathcal{T} ; \mathfrak{T}=\mathfrak{T}(\mathfrak{F}, \mathfrak{X}), \mathfrak{X} \in \mathcal{X}_{\mathfrak{F}}\right\} .
$$

Then we have

i) If $\mathfrak{T} \in \mathcal{T}$, then $\mathfrak{T} \in \mathcal{T}_{\mathfrak{F}}$ if and only if $\mathrm{b}(\mathfrak{T}) \subseteq \mathrm{b}(\mathfrak{F})$.

ii) The map

$$
\tau_{\mathfrak{F}}: \mathcal{X}_{\mathfrak{F}} \rightarrow \mathcal{T}_{\mathfrak{F}}
$$

given by $\tau_{\mathfrak{F}}(\mathfrak{X})=\mathfrak{T}(\mathfrak{F}, \mathfrak{X})$ if $\mathfrak{X} \in \mathcal{X}_{\mathfrak{F}}$ is a lattice antiisomorphism.

iii) $\mathcal{T}_{\mathfrak{F}}$ is a complete, distributive, complemented and atomic lattice.

Proof: i) By [1, XI 4.7], b(T) $\subseteq \mathrm{b}(\mathfrak{F})$ is equivalent to $\mathfrak{T}^{b} \subseteq \mathfrak{F} \subseteq \mathfrak{T}$.

By [2, Proposition 3], if $\mathfrak{T} \in \mathcal{T}_{\mathfrak{F}}$, then $\mathfrak{T}^{b} \subseteq \mathfrak{F} \subseteq \mathfrak{T}$. Conversely, if $\mathfrak{T} \in \mathcal{T}$ and $\mathfrak{T}^{b} \subseteq \mathfrak{F} \subseteq \mathfrak{T}$, set $\mathfrak{X}=\left(G \in \mathfrak{E} ; G=G_{\mathfrak{F}} G_{\mathfrak{M}}\right)$, where $\mathfrak{M}=\mathfrak{T}^{m}$. Then $\mathfrak{X} \in \mathcal{X}_{\mathfrak{F}}$ and $\mathfrak{T}=\mathfrak{T}(\mathfrak{F}, \mathfrak{X})$ by $[\mathbf{3}, 1.3,1.4]$, hence $\mathfrak{T} \in \mathcal{T}_{\mathfrak{F}}$.

ii) Consider the composition

$$
\mathcal{B}(\overline{\mathrm{b}}(\mathfrak{F})) \stackrel{\mathrm{f}}{\longrightarrow} \mathcal{X}_{\mathfrak{F}} \stackrel{\tau_{\mathfrak{F}}}{\longrightarrow} \mathcal{T}_{\mathfrak{F}}
$$

where $\mathrm{f}(\mathfrak{m})=\langle\mathfrak{F}, \mathfrak{m}\rangle$, if $\mathfrak{m} \subseteq \overline{\mathrm{b}}(\mathfrak{F})$. Then

$$
\begin{aligned}
\tau_{\mathfrak{F}} \mathrm{f}(\mathfrak{m}) & =\mathfrak{T}(\mathfrak{F},\langle\mathfrak{F}, \mathfrak{m}\rangle)=\left(G \in \mathfrak{E} ; G_{\mathfrak{F}} \mathrm{E}_{\mathfrak{m}}(G) \in \mathfrak{F}\right) \\
& =\left(G \in \mathfrak{E} ; \mathrm{E}_{\mathfrak{m}}(G) \in \mathfrak{F}\right)=\left(G \in \mathfrak{E} ; \mathbf{S}_{n}(G) \cap \mathfrak{m}=\emptyset\right) \\
& =\mathrm{h}(\mathfrak{m})
\end{aligned}
$$

hence $\tau_{\mathfrak{F}} \mathrm{f}$ is the restriction of $\mathrm{h}$ to $\mathcal{B}(\overline{\mathrm{b}}(\mathfrak{F}))$. Moreover if $\mathfrak{T} \in \mathcal{T}_{\mathfrak{F}}$, taking $\mathfrak{m}=\mathrm{b}(\mathfrak{T})$, we have that $\mathrm{h}(\mathfrak{m})=\mathfrak{T}$ and, by $\mathrm{i}), \mathfrak{m} \subseteq \overline{\mathrm{b}}(\mathfrak{F})$. Therefore $\tau_{\mathfrak{F}} \mathrm{f}$ is a bijection between $\mathcal{B}(\overline{\mathrm{b}}(\mathfrak{F}))$ and $\mathcal{T}_{\mathfrak{F}}$. 
Let $\mathfrak{T}, \mathfrak{R} \in \mathcal{T}_{\mathfrak{F}}$. Then, by i), $\mathfrak{T}^{b} \subseteq \mathfrak{F} \subseteq \mathfrak{R}$, and, by [1, XI 4.7], $\mathfrak{T} \subseteq \mathfrak{R}$ if and only if $\mathrm{b}(\mathfrak{R}) \subseteq \mathrm{b}(\mathfrak{T})$. Therefore $\tau_{\mathfrak{F}} \mathrm{f}$ is an antiisomorphism of ordered sets. Now, by Lemma 9 , we have that $f$ is an isomorphism, hence $\tau_{\mathfrak{F}}$ is also an antiisomorphism.

iii) That follows now immediately from Lemma 9.

Corollary 11. Let $\mathfrak{T}, \mathfrak{R} \in \mathcal{T}$. Then the following assertions are equivalent

i) There exists a Fitting class $\mathfrak{F}$ such that $\mathfrak{T}, \mathfrak{R} \in \mathcal{T}_{\mathfrak{F}}$.

ii) $\mathrm{b}(\mathfrak{T}) \cup \mathrm{b}(\mathfrak{R})$ is a preboundary.

iii) $b(\mathfrak{T}) \cup b(\mathfrak{R})=b(\mathfrak{T} \cap \mathfrak{R})$.

Proof: i) $\Longrightarrow$ iii) by Theorem 10. Of course iii) $\Longrightarrow$ ii). If $\mathrm{b}(\mathfrak{T}) \cup \mathrm{b}(\mathfrak{R})$ is a preboundary, as it consists of perfect groups, then, by [1, XI 4.3],

$$
\mathfrak{H}=\mathrm{h}(\mathrm{b}(\mathfrak{T}) \cup \mathrm{b}(\mathfrak{R}))
$$

is a Fitting class (incidentally belonging to $\mathcal{T}$ ) such that $\mathrm{b}(\mathfrak{H})=\mathrm{b}(\mathfrak{T}) \cup$ $\mathrm{b}(\mathfrak{R})$. In particular $\mathrm{b}(\mathfrak{T}) \subseteq \mathrm{b}(\mathfrak{H})$ and $\mathrm{b}(\mathfrak{R}) \subseteq \mathrm{b}(\mathfrak{H})$, hence $\mathfrak{T}, \mathfrak{R} \in \mathcal{T}_{\mathfrak{H}}$, by Theorem 10, and we have finally that ii) $\Longrightarrow$ i).

Remarks 12. Let $\mathfrak{F}$ be a Fitting class.

i) Obviously $\max \left(\mathcal{T}_{\mathfrak{F}}\right)=\mathfrak{E}$. On the other hand, $\min \left(\mathcal{T}_{\mathfrak{F}}\right)=\mathrm{h}(\overline{\mathrm{b}}(\mathfrak{F}))$ by Theorem 10. And it is immediate that

$$
\mathrm{h}(\overline{\mathrm{b}}(\mathfrak{F}))=\left(G ; \operatorname{Soc}_{n}\left(G / G_{\mathfrak{F}}\right)=1\right) .
$$

ii) $\mathcal{T}_{\mathfrak{F}}$ is not a sublattice of $\mathcal{T}$. If $\mathfrak{T}, \mathfrak{R} \in \mathcal{T}_{\mathfrak{F}}$, then $\mathrm{b}(\mathfrak{T} \cap \mathfrak{R})=\mathrm{b}(\mathfrak{T}) \cup$ $\mathrm{b}(\mathfrak{R})$, by Corollary 11 , hence the infimum of two elements in $\mathcal{T}_{\mathfrak{F}}$ coincides with that in $\mathcal{T}$. But the question changes for the supremum: it suffices to consider that $\mathcal{T}_{\mathfrak{F}}$ is complemented and Remark 2 (i).

In fact, for all $\mathfrak{T}, \mathfrak{R} \in \mathcal{T}, \mathrm{b}(\mathfrak{T}) \cap \mathrm{b}(\mathfrak{R}) \subseteq \mathrm{b}(\langle\mathfrak{T}, \mathfrak{R}\rangle \mathfrak{S})$, with equality if and only if $\mathrm{b}(\mathfrak{T}) \subseteq \mathrm{b}(\mathfrak{R})$ or $\mathrm{b}(\mathfrak{R}) \subseteq \mathrm{b}(\mathfrak{T})$.

To see it, let us assume first that $\mathrm{b}(\langle\mathfrak{T}, \mathfrak{R}\rangle \mathfrak{S}) \subseteq \mathrm{b}(\mathfrak{T}) \cap \mathrm{b}(\mathfrak{R})$ and suppose moreover that $\mathfrak{T} \not \mathfrak{R} \nsubseteq \mathfrak{T}$. Take $X \in \mathfrak{R} \cap \mathrm{b}(\mathfrak{T}), Y \in \mathfrak{T} \cap \mathrm{b}(\mathfrak{R})$ and $W$ as in Remark $2(\mathrm{i})$; then $W \in \mathrm{b}(\langle\mathfrak{T}, \mathfrak{R}\rangle \mathfrak{S}) \backslash(\mathrm{b}(\mathfrak{T}) \cap \mathrm{b}(\mathfrak{R}))$, against the hypothesis. We may assume therefore that $\mathfrak{T} \subseteq \mathfrak{R}$. Then $\langle\mathfrak{T}, \mathfrak{R}\rangle \mathfrak{S}=$ $\mathfrak{R}$, hence $\mathrm{b}(\mathfrak{R}) \subseteq \mathrm{b}(\mathfrak{T}) \cap \mathrm{b}(\mathfrak{R})$ and so $\mathrm{b}(\mathfrak{R}) \subseteq \mathrm{b}(\mathfrak{T})$.

Let us assume now that $\mathrm{b}(\mathfrak{R}) \subseteq \mathrm{b}(\mathfrak{T})$. Then $\mathfrak{T} \subseteq \mathfrak{R}$ by [1, XI 4.4], hence $\mathrm{b}(\langle\mathfrak{T}, \mathfrak{R}\rangle \mathfrak{S})=\mathrm{b}(\mathfrak{R}) \subseteq \mathrm{b}(\mathfrak{T}) \cap \mathrm{b}(\mathfrak{R})$.

iii) If $\bar{b}(\mathfrak{F})=\mathfrak{m} \cup \mathfrak{n}$ and $\mathfrak{m} \cap \mathfrak{n}=\emptyset$, then $\mathfrak{T}=\mathrm{h}(\mathfrak{m})$ and $\mathfrak{R}=\mathrm{h}(\mathfrak{n})$ are mutual complements in $\mathcal{T}_{\mathfrak{F}}, \mathfrak{T}^{m}=\langle\mathfrak{m}\rangle$ and $\mathfrak{R}^{m}=\langle\mathfrak{n}\rangle$. Obviously 
$\mathfrak{m} \subseteq \mathfrak{R}^{\mathfrak{s}}$ and $\mathfrak{n} \subseteq \mathfrak{T}^{\mathfrak{s}}$. In fact, $\langle\mathfrak{m}\rangle \subset \mathfrak{R}^{\mathfrak{s}}$ and $\langle\mathfrak{n}\rangle \subset \mathfrak{T}^{\mathfrak{s}}$. For if $X \in \mathfrak{m}$, take any $S \in \Sigma$ and suppose that $X \imath S \notin \mathfrak{R}$; then $X \imath S \in \mathrm{b}(\mathfrak{R})=\mathfrak{n}$, as $X \imath S$ is perfect and single-headed with cosocle in $\mathfrak{R}$, by [1, A 18.8], which contradicts that $\mathfrak{m} \cup \mathfrak{n}$ is subnormally independent; therefore $X \imath S \in \mathfrak{R}^{\mathfrak{s}}$; but $X\langle S \notin\langle\mathfrak{m}\rangle$ by [1, XI 4.14] because $\mathfrak{m}$ is subnormally independent. Analogously $\langle\mathfrak{n}\rangle \subset \mathfrak{T}^{\mathfrak{s}}$. In particular, $\mathfrak{T}^{m} \subset \mathfrak{R}_{*}$ and $\mathfrak{R}^{m} \subset \mathfrak{T}_{*}$, by $[\mathbf{1}$, XI 4.15].

iv) By Theorem 10 (i) and [2, Proposition 13], there exists $\mathfrak{T} \in \mathcal{T}_{\mathfrak{F}}$ such that $\mathfrak{T}^{b}=\mathfrak{T}_{*}$ if and only if $\overline{\mathrm{b}}(\mathfrak{F})$ is maximal in $(\mathcal{P}, \subseteq)$. In this case such a $\mathfrak{T}$ is unique, namely $\mathfrak{T}=\mathrm{h}(\overline{\mathrm{b}}(\mathfrak{F}))=\mathfrak{F} \mathfrak{S}=\mathfrak{F}^{*}$; moreover, $\mathfrak{F} \in \operatorname{Locksec}(\mathfrak{T})$, hence $\mathfrak{F}$ is injective, by $[\mathbf{3}, 3.4]$.

v) If $\mathfrak{F}$ and $\mathfrak{G}$ are two Fitting classes, then $\mathcal{T}_{\mathfrak{F}}=\mathcal{T}_{\mathfrak{G}}$ if and only if $\overline{\mathrm{b}}(\mathfrak{F})=\overline{\mathrm{b}}(\mathfrak{G})$, by Theorem 10 (i). Therefore $\mathcal{T}_{\mathfrak{F}}=\mathcal{T}_{\mathrm{h}(\overline{\mathrm{b}}(\mathfrak{F}))}$.

In particular, by [2, Proposition 13], if $\mathfrak{T} \in \mathcal{T}$ satisfies $\mathfrak{T}^{b}=\mathfrak{T}_{*}$, then there is a unique $\mathcal{E} \in\left\{\mathcal{T}_{\mathfrak{F}} ; \mathfrak{F}\right.$ is a Fitting class $\}$ such that $\mathfrak{T} \in \mathcal{E}$, namely $\mathcal{E}=\mathcal{T}_{\mathfrak{T}}$

In general, given $\mathfrak{T} \in \mathcal{T}$, the set of lattices $\mathcal{T}_{\mathfrak{F}}$ according to Theorem 10 for which $\mathfrak{T} \in \mathcal{T}_{\mathfrak{F}}$ is

$$
\left\{\mathcal{T}_{\mathfrak{H}} ; \mathfrak{H} \in \mathcal{T}, \mathfrak{T}^{b} \subseteq \mathfrak{H} \subseteq \mathfrak{T}\right\}
$$

by $\left[\mathbf{1}\right.$, XI 4.7]. Observe that if $\mathfrak{T} \in \mathcal{T}_{\mathfrak{H}}, \mathfrak{H} \in \mathcal{T}$, then the complement of $\mathfrak{T}$ in $\mathcal{T}_{\mathfrak{H}}$ is

$$
\mathfrak{R}=\mathrm{h}(\mathrm{b}(\mathfrak{H}) \backslash \mathrm{b}(\mathfrak{T}))=\mathrm{h}(\mathrm{b}(\mathfrak{H}) \cap \mathfrak{T}) .
$$

We have $\mathfrak{T} \cap \mathfrak{R}=\min \left(\mathcal{T}_{\mathfrak{H}}\right)=\mathfrak{H}$. On the other hand if $\mathfrak{G}$ is a Fitting class such that $\mathfrak{T} \cap \mathfrak{G} \subseteq \mathfrak{H}$, then

$$
\mathfrak{G} \cap \mathrm{b}(\mathfrak{R})=\mathfrak{G} \cap \mathrm{b}(\mathfrak{H}) \cap \mathfrak{T} \subseteq \mathfrak{H} \cap \mathrm{b}(\mathfrak{H})=\emptyset,
$$

hence $\mathfrak{G} \subseteq \mathfrak{R}$. Therefore $\mathfrak{R}$ is the greatest Fitting class $\mathfrak{G}$ such that $\mathfrak{T} \cap \mathfrak{G} \subseteq \mathfrak{H}$.

If $\mathfrak{H}, \mathfrak{K} \in \mathcal{T}, \mathfrak{T}^{b} \subseteq \mathfrak{H}, \mathfrak{K} \subseteq \mathfrak{T}$ and $\mathfrak{R}$, resp. $\mathfrak{L}$, is the complement of $\mathfrak{T}$ in $\mathcal{T}_{\mathfrak{H}}$, resp. $\mathcal{T}_{\mathfrak{K}}$, then $\mathfrak{H} \subseteq \mathfrak{K}$ if and only if $\mathfrak{R} \subseteq \mathfrak{L}$.

Indeed if $\mathfrak{H} \subseteq \mathfrak{K}$, then $\mathfrak{R} \cap \mathfrak{T}=\mathfrak{H} \subseteq \mathfrak{K}$, hence $\mathfrak{R} \subseteq \mathfrak{L}$. And if $\mathfrak{R} \subseteq \mathfrak{L}$, then $\mathfrak{H} \cap \mathrm{b}(\mathfrak{K})=\mathfrak{T} \cap \mathfrak{R} \cap \mathrm{b}(\mathfrak{K}) \subseteq \mathfrak{T} \cap \mathfrak{L} \cap \mathrm{b}(\mathfrak{K})=\mathfrak{K} \cap \mathrm{b}(\mathfrak{K})=\emptyset$, hence $\mathfrak{H} \subseteq \mathfrak{K}$.

Example 13. Let $\mathfrak{N}$ be the class of nilpotent groups and consider the class

$$
\mathfrak{C}=\left(G ; \mathrm{F}(G)=\mathrm{F}^{*}(G)\right)
$$

of $\mathfrak{N}$-constrained groups. Then $\mathfrak{C}=\mathfrak{T}(\mathfrak{N}, \widetilde{\mathfrak{N}})$, where $\tilde{\mathfrak{N}}=(G ; G=$ $\left.\mathrm{F}^{*}(G)\right)$ is the class of generalized nilpotent groups (see [1, IX 4.14]). We noted in $\left[\mathbf{2}\right.$, Example (iii)] that $\mathfrak{C}^{b}=\mathfrak{N}$ and $\mathfrak{C}^{m}=\widetilde{\mathfrak{N}}$. 
Let $\mathfrak{F}$ be a Fitting class such that $\mathfrak{C} \in \mathcal{T}_{\mathfrak{F}}$. The complement of $\mathrm{b}(\mathfrak{C})$ in the boolean $\mathcal{B}(\overline{\mathrm{b}}(\mathfrak{F}))$ is

$$
\mathfrak{n}=(X \in \overline{\mathrm{b}}(\mathfrak{F}) ; \mathrm{Z}(X)<\operatorname{Cos}(X))
$$

By Lemma 9 and Theorem 10, the complement of $\mathfrak{C}$ in $\mathcal{T}_{\mathfrak{F}}$ is $\mathfrak{R}=\mathrm{h}(\mathfrak{n})$. Let us see that $\mathfrak{N} \subseteq \mathfrak{R}^{b}$. Let $p$ be a prime, take $S \in \Sigma$ such that $p$ is a divisor of $n=|S|$ and consider the regular wreath product $W=C_{p} \imath S$. Set $X=W^{\prime}$. From $[1, \mathrm{~A} 18.4]$, with $M=\left\{\left(x_{1}, \ldots, x_{n}\right) \in C_{p}^{n} ; x_{1} \ldots x_{n}=\right.$ $1\}$, we have that $X=M S$ is perfect and single-headed; $\mathrm{Z}(X)$ is the diagonal subgroup of $C_{p}^{n}$ and, as $p \mid n$, we have that $C_{p} \cong \mathrm{Z}(X)<M=$ $\operatorname{Cos}(X)$, hence $X \in \mathfrak{n}, C_{p} \in\langle\operatorname{Cos}(G) ; G \in \mathfrak{n}\rangle=\mathfrak{R}^{b}$, and $\mathfrak{N} \subseteq \mathfrak{R}^{b}$.

Consider the case $\mathfrak{F}=\mathfrak{N}$. We have that

$\overline{\mathrm{b}}(\mathfrak{N})=(X ; X$ is perfect and single-headed and $\operatorname{Cos}(X)=\mathrm{F}(X))$.

Now obviously $\langle\operatorname{Cos}(G) ; G \in \mathfrak{n}\rangle \subseteq \mathfrak{N}$, hence $\mathfrak{R}^{b}=\mathfrak{N}$ and $\mathfrak{R}^{m}=$ $\langle\mathfrak{n}\rangle=\left(G ; G=\mathrm{F}(G) \mathrm{E}_{\mathfrak{n}}(G)\right)$, by [2, Proposition 7]. On the other hand $\langle\mathfrak{N}, \mathrm{b}(\mathfrak{C})\rangle=\langle\mathrm{b}(\mathfrak{C})\rangle=\tilde{\mathfrak{N}}$ and, by Lemma $9, \widetilde{\mathfrak{N}}$ and $\langle\mathfrak{n}\rangle$ are mutual complements in $\mathcal{X}_{\mathfrak{N}}$. Therefore $\tilde{\mathfrak{N}} \cap\langle\mathfrak{n}\rangle=\mathfrak{N}$ and

$$
\widetilde{\mathfrak{N}} \cdot\langle\mathfrak{n}\rangle=\left(G ; G=\mathrm{F}^{*}(G) G_{\langle\mathfrak{n}\rangle}\right)=\mathfrak{N} \diamond \mathbf{D}_{0} \Sigma .
$$

Thus for each group $G \in \mathfrak{E}$ we have

$$
\mathrm{F}^{*}(G) / \mathrm{F}(G) \times G_{\langle\mathfrak{n}\rangle} / \mathrm{F}(G)=(G / \mathrm{F}(G))_{\mathbf{D}_{0} \Sigma},
$$

the nonabelian socle of $G / \mathrm{F}(G)$. Moreover

$$
\min \mathcal{T}_{\mathfrak{N}}=\left(G ; \operatorname{Soc}_{n}(G / \mathrm{F}(G))=1\right)=\mathfrak{T}\left(\mathfrak{N}, \mathfrak{N} \diamond \mathbf{D}_{0} \Sigma\right)=\mathrm{h}(\overline{\mathrm{b}}(\mathfrak{N})) .
$$

By $[\mathbf{2}$, Proposition 3 (ii)] it follows that

$$
\mathfrak{R}=\mathrm{h}(\mathfrak{n})=\mathfrak{T}(\mathfrak{N},\langle\mathfrak{n}\rangle)=\left(G ; G=\mathrm{C}_{G}\left(G_{\langle\mathfrak{n}\rangle} / \mathrm{F}(G)\right)\right)
$$

is the complement of $\mathfrak{C}$ in $\mathcal{T}_{\mathfrak{N}}$. Observe that $\mathfrak{C}^{b}=\mathfrak{N}=\mathfrak{R}^{b} \subseteq \mathfrak{R}^{m} \subseteq \mathfrak{C}^{\mathfrak{s}}$, hence $\mathfrak{C}_{*}=\mathfrak{C}^{\mathfrak{s}}$ by $[\mathbf{3}, 1.1]$. Analogously $\mathfrak{R}_{*}=\mathfrak{R}^{\mathfrak{s}}$.

Assume now that $\mathfrak{F} \in \operatorname{Locksec}(\mathfrak{S})$, that is, $\mathfrak{S}_{*} \subseteq \mathfrak{F} \subseteq \mathfrak{S}$. As $\mathfrak{S}_{*}=\mathfrak{S}^{b}$, this is equivalent to $\mathrm{b}(\mathfrak{S}) \subseteq \mathrm{b}(\mathfrak{F})$, by $[\mathbf{1}, \mathrm{XI} 4.7]$, and then, immediatly, to $\mathrm{b}(\mathfrak{S})=\overline{\mathrm{b}}(\mathfrak{F})$. Let us see that in this case $\mathfrak{R}^{b}=\mathfrak{S}_{*}$. Let $X \in \mathrm{b}(\mathfrak{S})$ and set $Y=\operatorname{Cos}(X)$. If $Y=\mathrm{Z}(X)$, in particular $Y \in \mathfrak{N} \subseteq \mathfrak{R}^{b}$. If $\mathrm{Z}(X)<Y$, then $X \in \mathfrak{n}$, hence $Y \in \mathfrak{R}^{b}$. Therefore $\mathfrak{S}^{b} \subseteq \mathfrak{R}^{b}$. On the other hand, $\mathrm{b}(\mathfrak{R})=\mathfrak{n} \subseteq \mathrm{b}(\mathfrak{S})$, hence $\mathfrak{R}^{b} \subseteq \mathfrak{S}^{b}$ and equality holds. Let us see further that $\min \mathcal{T}_{\mathfrak{F}}=\mathfrak{S}$. If we assume the contrary, then there

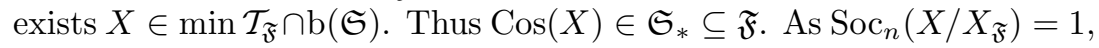
it follows $X \in \mathfrak{F}$, against $\mathfrak{F} \subseteq \mathfrak{S}$. Hence in fact $\min \mathcal{T}_{\mathfrak{F}}=\mathfrak{S}$. Observe in particular that $\max \mathcal{X}_{\mathfrak{S}_{*}}=\left\langle\mathfrak{S}_{*}, \mathrm{~b}(\mathfrak{S})\right\rangle=\langle\mathrm{b}(\mathfrak{S})\rangle=\mathfrak{S}_{*} \diamond \mathbf{D}_{0} \Sigma$, the smallest normal Fitting class, and that $\max \mathcal{X}_{\mathfrak{S}}=\langle\mathfrak{S}, \mathrm{b}(\mathfrak{S})\rangle=\mathfrak{S} \diamond \mathbf{D}_{0} \Sigma$, 
the class of all groups $G$ which induce an inner automorphism on each nonabelian chief factor of $G$, which is also a normal Fitting class by [1, $\mathrm{X} 3.28]$, as $\mathfrak{S} \diamond\left(\mathfrak{S}_{*} \diamond \mathbf{D}_{0} \Sigma\right)=\mathfrak{S} \diamond \mathbf{D}_{0} \Sigma$.

In $[\mathbf{3}, 2.8-9]$ we saw that $\mathcal{B L}=\{\langle\mathfrak{T}, \mathrm{b}(\mathfrak{T})\rangle ; \mathfrak{T} \in \mathcal{T}\}$ is exactly the set of normal Fitting classes defined by Blessenohl and Laue [1, X 3.20]. Consider $\mathcal{B} \mathcal{L}$ ordered by the inclusion relation.

Theorem 14. The map

$$
\nu: \mathcal{T} \rightarrow \mathcal{B L}
$$

given by $\nu(\mathfrak{T})=\langle\mathfrak{T}, \mathrm{b}(\mathfrak{T})\rangle$ is an isomorphism of ordered sets. In particular $\mathcal{B L}$ is a complete, distributive and atomic lattice.

Proof: If $\mathfrak{T}, \mathfrak{R} \in \mathcal{T}$ and $\mathfrak{T} \subseteq \mathfrak{R}$, then immediatly

$$
\mathrm{b}(\mathfrak{T})=(\mathrm{b}(\mathfrak{R}) \cap \mathrm{b}(\mathfrak{T})) \cup(\mathfrak{R} \cap \mathrm{b}(\mathfrak{T})) .
$$

Therefore $\nu(\mathfrak{T}) \subseteq \nu(\mathfrak{R})$

Assume now that $\langle\mathfrak{T}, \mathrm{b}(\mathfrak{T})\rangle \subseteq\langle\mathfrak{R}, \mathrm{b}(\mathfrak{R})\rangle$, where $\mathfrak{T}, \mathfrak{R} \in \mathcal{T}$. We see that $\mathfrak{T} \subseteq \mathfrak{R}$. Assume the contrary and let $X \in \mathfrak{T} \cap \mathrm{b}(\mathfrak{R})$. Take $S \in \Sigma$ and consider $Y=X \imath S . Y$ is a perfect and single-headed group with $\operatorname{Cos}(Y) \in \mathfrak{T}$, hence either $Y \in \mathfrak{T}$ or $Y \in \mathrm{b}(\mathfrak{T})$. In any case $Y \in\langle\mathfrak{T}, \mathrm{b}(\mathfrak{T})\rangle$, hence $Y \in\langle\mathfrak{R}, \mathrm{b}(\mathfrak{R})\rangle$. By $[\mathbf{1}, \mathrm{XI} 4.14]$, we have $Y \in \mathfrak{R}$ or $Y \in \mathrm{b}(\mathfrak{R})$, in contradiction with $X$ sn $Y, X \neq Y$ and $X \in \mathrm{b}(\Re)$.

\section{References}

[1] K. Doerk AND T. HAWkes, "Finite soluble groups", de Gruyter Expositions in Mathematics 4, Walter de Gruyter \& Co., Berlin, 1992.

[2] M. J. Iranzo, J. P. Lafuente and F. PÉrez-Monasor, Preboundaries of perfect groups, J. Group Theory 6(1) (2003), 57-68.

[3] M. J. Iranzo, J. P. Lafuente and F. PÉrez-Monasor, Preboundaries of perfect groups II, J. Group Theory 7(1) (2004), $113-125$.

[4] V. D. Mazurov and E. I. Khukhro, Eds., "The Kourovka notebook. Unsolved problems in group theory", Fourteenth augmented edition, Russian Academy of Sciences Siberian Division, Institute of Mathematics, Novosibirsk, 1999. 
M. J. Iranzo:

Departamento de Álgebra

Universidad de Valencia

c/ Dr. Moliner, 50

46100 Burjasot (Valencia)

Spain

E-mail address: M.Jesus.Iranzo@uv.es

Julio P. Lafuente:

Departamento de Matemática e Informática

Universidad Pública de Navarra

Campus Arrosadía s/n

31006 Pamplona (Navarra)

Spain

E-mail address: lafuente@unavarra.es

F. Pérez-Monasor:

Departamento de Álgebra

Universidad de Valencia

c/ Dr. Moliner, 50

46100 Burjasot (Valencia)

Spain

E-mail address: F.Perez.Monasor@uv.es

Rebut el 7 d'octubre de 2004. 